\title{
Structural Diversity of Photoswitchable Sphingolipids for Optodynamic Control of Lipid Raft Microdomains
}

\author{
Nina Hartrampf ${ }^{1,2, \ddagger}$, Samuel M. Leitao ${ }^{3,4, \mp}$, Nils Winter ${ }^{1}$, Henry Toombs-Ruane ${ }^{1}$, \\ James A. Frank ${ }^{1,5}$, Petra Schwille ${ }^{3}$, Dirk Trauner ${ }^{1,6, *}$, Henri G. Franquelim ${ }^{3, *}$
}

\begin{abstract}
1) Department of Chemistry, Ludwig Maximilian University of Munich, Butenandtstraße 5-13, Munich 81377, Germany; 2) Department of Chemistry, University of Zurich, Winterthurerstrasse 190, Zurich 8057, Switzerland; 3) Cellular and Molecular Biophysics Department, Max Planck Institute of Biochemistry, Am Klopferspitz 18, Martinsried 82152, Germany; 4) Institute for Bioengineering, École Polytechnique Fédérale de Lausanne, Bâtiment BM 3109 Station 17, Lausanne 1015, Switzerland; 5) Vollum Institute, Oregon Health \& Science University, 3181 SW Sam Jackson Park Road, Portland, Oregon 97239, United States; 6) Department of Chemistry, New York University, 100 Washington Square East, New York City, New York 10003, United States.
\end{abstract}

\footnotetext{
$¥$ Co-authors with equal contribution

*Corresponding authors: HGF (hgfranq@biochem.mpg.de) \& DT (dirktrauner@nyu.edu)
}

\begin{abstract}
Sphingolipids are a structurally diverse class of lipids predominantly found in the plasma membrane of eukaryotic cells. These lipids can laterally segregate with other saturated lipids and cholesterol into lipid rafts; liquid-ordered $\left(L_{\circ}\right)$ microdomains that act as organizing centers within biomembranes. Owing the vital role of sphingolipids for lipid segregation, controlling their lateral localization is of utmost significance. Hence, we made use of the light-induced trans-cis isomerization of azobenzene-modified acyl chains, to develop a set of photoswitchable sphingolipids, with different headgroups (hydroxyl, galactosyl, phosphocholine) and backbones (sphingosine, phytosphingosine, tetrahydropyran (THP)blocked sphingosine), able to shuttle between liquid-ordered $\left(L_{o}\right)$ and liquid-disordered $\left(L_{d}\right)$ regions of model membranes upon irradiation with UV-A $(\lambda=365 \mathrm{~nm})$ and blue $(\lambda=470 \mathrm{~nm})$ light, respectively. Using combined high-speed atomic force microscopy, fluorescence microscopy, and force spectroscopy, we investigated how these active sphingolipids laterally remodel supported bilayers upon photo-isomerization, notably in terms of domain area changes, height mismatch, line tension, and membrane piercing. Hereby, we show that all

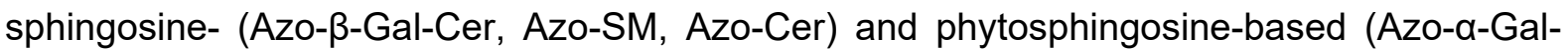
PhCer, Azo-PhCer) photolipids behave similarly, promoting a reduction in $\mathrm{L}_{\circ}$ domain area when in the UV-adapted cis-isoform. In contrast, azo-sphingolipids having THP groups that block $\mathrm{H}$-bonding at the sphingosine backbone (Azo-THP-SM, Azo-THP-Cer) induce an increase in the $L_{o}$ domain area when in cis, accompanied by a major rise in height mismatch and line tension. These changes were fully reversible upon blue light-triggered isomerization of the various lipids back to trans, pinpointing the role of interfacial interactions for the formation of stable $L_{o}$ lipid raft domains.
\end{abstract}




\section{Introduction}

Sphingolipids are a major component of eukaryotic (notably mammalian) membranes and play a crucial role in signaling inside cells ${ }^{[1-3]}$. Members of this lipid class, like ceramide (Cer) or sphingomyelin (SM), have a backbone formed from sphingosine, and have been widely studied in terms of their biophysical properties, behavior on membrane models, and affinity to other lipids ${ }^{[4-11]}$. Another important, but less studied, class of sphingolipids are phytosphingolipids, which are abundant in plants and fungi ${ }^{[12-14]}$. These lipids have phytosphingosine as sphingoid base ${ }^{[2]}$, a backbone with increased polarity in comparison to sphingosine. Sphingolipids are also largely localized in the plasma membranes of eukaryotic cells, when compared to inner organelle membranes ${ }^{[3]}$. Here, they are usually linked to socalled lipid rafts ${ }^{[15]}$, liquid ordered $\left(L_{0}\right)$ phase nano- or microdomains composed of saturated lipid species and sterols, thought to be a means by which cells organize or segregate important proteins within the membrane ${ }^{[16-18]}$.

From a molecular point of view, sphingolipids can form stable hydrogen-bond and hydrophobic interactions with other sphingolipids (e.g. SM) and cholesterol $(\mathrm{Chol})^{[2,19]}$. This can, e.g., be observed in vitro on supported membrane model systems, as segregated rigid $L_{o}$ phase microdomains embedded in a fluid bulk liquid disordered $\left(L_{d}\right)$ membrane phase ${ }^{[20-24]}$. The presence of hydroxyl $(-\mathrm{OH})$ groups on the backbone of sphingolipids are particularly relevant for the formation of $\mathrm{L}_{\circ}$ domains, as interfacial $\mathrm{H}$-bonding markedly stabilize the interactions among sphingolipids and Chol[19,25]. In fact, the central role of the $3-\mathrm{OH}$ moiety on the sphingosine backbone of sphingolipids, like Cer or SM, has been thoroughly scrutinized[13,2632]. Likewise, the presence of a second 4-OH hydroxyl group on phytosphingosine-based lipids, like $\mathrm{PhCer}$, further strengthens $\mathrm{H}$-bonding and domain thermostability ${ }^{[13]}$. In contrast, hindering $\mathrm{H}$-bonding by adding a methyl, ethyl or tetrahydropyranyl (THP) group at the 3-OH hydroxyl, severely affected the molecular packing and ability of those blocked sphingolipids to interact with Chol[26,33]. Indeed, functionalization of the 3-OH of SM by THP greatly decreased gel-phase stability (lowering the melting temperature $\left(T_{\mathrm{m}}\right)$ by $10^{\circ} \mathrm{C}$ ), impede tight contacts with Chol, and increase the rate of sterol desorption from vesicles containing this blocked SM analog ${ }^{[26]}$.

As lipid segregation plays a crucial physiological role in biomembranes, new nano-tools to investigate and control membrane phase properties are urgently needed. In that regard, strategies based on photopharmacology[34,35], which take advantage of the high spatiotemporal precision of light, are particularly appealing. In 2016, we reported that photoswitchable ceramides (ACes), which have an azobenzene photoswitch incorporated in the lipid fatty acid chain, enable optical control of lipid rafts within synthetic membranes. ${ }^{[36]}$ While recent advancements already demonstrated the potential of azobenzene-modified photoswitchable lipids for altering membrane properties ${ }^{[36-43]}$, the structural diversity of these photo-active molecules is still fairly limited. This stands in stark contrast with the impressive diversity of sphingolipids found in nature. Indeed, diverse sphingolipids can serve as docking site for various toxins (e.g. Shiga toxin binds to $\mathrm{Gb} 3^{[67]}$ or Cholera toxin B binds to $\mathrm{GM}{ }^{[44]}$ ), or even modulate the uptake of viruses by the cells (e.g. SV40 requires GM1 as receptor ${ }^{[45]}$ or HIV-1 gp120 surface protein binds to GalCer on epithelial cells $\left.{ }^{[46]}\right)$. Hence, controlling their lateral localization within membranes is of vital importance. An expanded palette of photoswitchable sphingolipids could therefore offer new photo-responsive $N$-acyl azobenzene sphingolipids with more complex headgroup functionalities and other types of sphingoid backbones. 
In our present work we then aimed to develop a new set of photoswitchable sphingolipids with increased functionalization and incorporate these various photolipids into $L_{d}-L_{o}$ phaseseparated supported model membranes. Our main goal was to investigate the influence of these modifications on the reversible remodeling of membranes microdomains upon photoswitching, as well as on fundamental mechanical properties of lipid bilayers. To this end, we performed atomic force microscopy (AFM) combined with fluorescence confocal microscopy, following the generated changes in domain area, height and line tension.

A<smiles>CCCCCCCCCCCC=CC(C)CO</smiles>

Sphingosine base (3-OH + 4-trans)<smiles>CCCCCCCCCCCCC(O)C(C)CO</smiles>

Phytosphingosine base $(3-\mathrm{OH}+4-\mathrm{OH})$

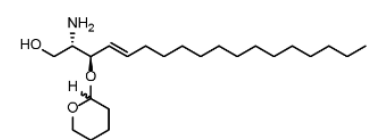

Blocked sphingosine base (3-OTHP + 4-trans)

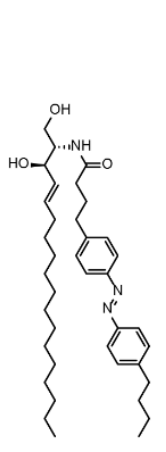

AzoCer

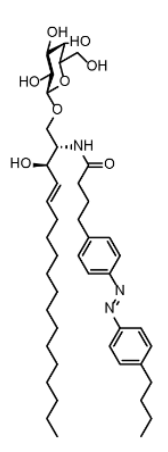

Azo$\beta$-Gal-Cer

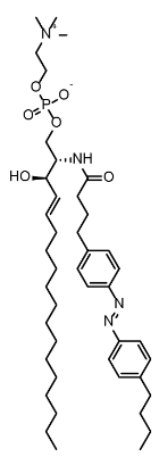

Azo-

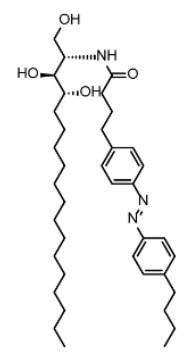

Azo-

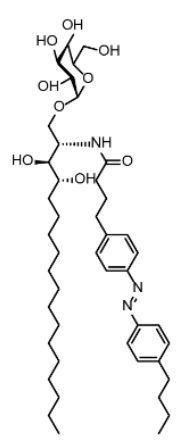

Azo$\alpha-G a l-P h C e r$

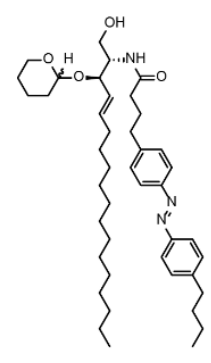

AzoTHP-Cer

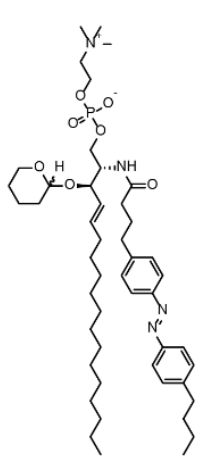

AzoTHP-SM
B

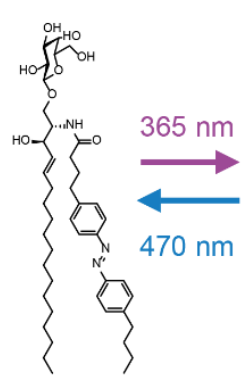

C

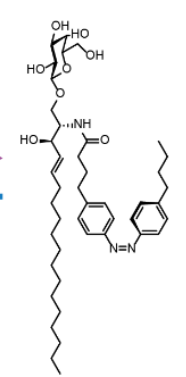

Azo- $\beta$-Gal-Cer

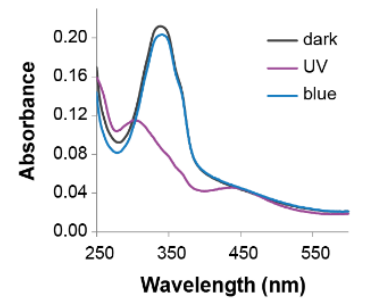

Azo-a-Gal-PhCer

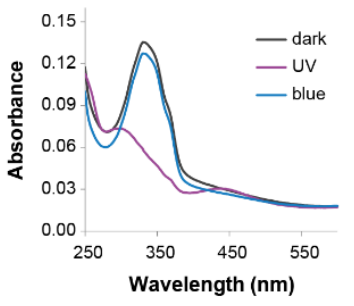

Azo-THP-SM

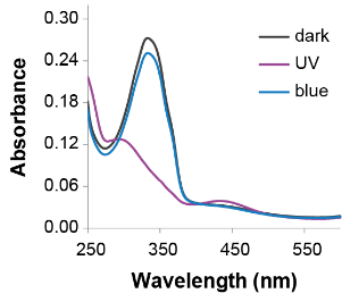

Fig. 1 - Structural and spectral properties of photoswitchable azo-sphingolipids. (A) chemical structures of the $\mathrm{N}$-acyl azobenzene-modified (FAazo-4 fatty acid) sphingolipids here tested, subdivided according to their sphingoid backbone: Azo-Cer, Azo- $\beta$-Gal-Cer and Azo-SM with a sphingosine base, Azo-PhCer and Azo- $\alpha$-Gal-PhCer with a phytosphingosine base, as well as Azo-THP-Cer and AzoTHP-SM displaying a 3-OH-blocked sphingosine base with a THP protecting group. (B) Schematics of light-induced trans-cis isomerization for an azo-sphingolipid, notably Azo- $\beta$-Gal-Cer. Application of UVA light $(\lambda=365 \mathrm{~nm})$ leads to the formation of cis-photolipid, while illumination with blue light $(\lambda=470 \mathrm{~nm})$ leads to the formation of trans-photolipid. (C) UV-Vis absorbance spectra of photoswitchable sphingolipids (notably Azo- $\beta$-Gal-Cer, Azo- $\alpha$-Gal-PhCer and Azo-THP-SM) incorporated in SUVs, at the dark-adapted state (black curves), as well as after the sequential shining of UV-A (purple curves) and blue light (blue curves). 


\section{Results and Discussion}

\subsection{Synthesis of photoswitchable sphingolipids}

Inspired by the structural design of our simpler azo-ceramides (ACes) ${ }^{[36]}$ and more complex a-galactosyl-phytoceramides ( $\alpha$-GalACers) $)^{[37]}$, we introduced five new azobenzene-modified sphingolipids, namely Azo-PhCer, Azo-THP-Cer, Azo- $\beta$-Gal-Cer, Azo-SM and Azo-THPSM. These photolipids featured (Fig. 1A): 1) a FAAzo-4 fatty acid ${ }^{[34]}$ at the $N$-acyl chain (equivalent to a $\Delta 9$ unsaturation when in the cis-isoform), 2) sphingoid backbones based on naturally-occurring sphingosine and phytosphingosine, or hydroxyl-blocked sphingosine with a THP protecting group, as well as 3) lipid headgroups presenting either a free $-\mathrm{OH}$, galactosyl or phosphocholine moiety. For our comparative studies, Azo-Cer (previously named ACe1) ${ }^{[36]}$ and Azo- $\alpha-G a l-P h C e{ }^{[37]}$ (previously named GalACer-4), having the same FAAzo-4 moiety able to undergo trans-cis photoisomerization (Fig. 1B), were also assessed.

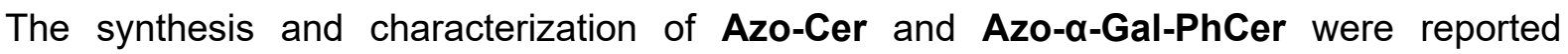
elsewhere ${ }^{[36,37]}$. Azo-PhCer was prepared analogously to Azo-Cer by the coupling of phytoceramide with FAAzo-4 using HBTU as a coupling agent (see SI). Additional protecting group manipulations yielded Azo-THP-Cer.

For the synthesis of Azo- $\boldsymbol{\beta}-\mathbf{G a l}-\mathbf{C e r}$, we used a benzoyl protected alcohol and the azide as protecting groups $^{[47]}$ (see SI). Azides do not coordinate to the primary alcohol and thereby the nucleophilicity of the sphingosine is greatly enhanced. Glycosylation of azidosphingosine with trichloroacetimidate yielded protected glycoside in $92 \%$ yield and excellent $\beta$-selectivity. Staudinger reduction using $\mathrm{PBu}_{3}$ and subsequent amide coupling with $\mathrm{FAAzo}-4^{[34]}$ using 1ethyl-3-(3-dimethylaminopropyl)carbodiimide (EDCI), followed by global deprotection gave Azo- $\beta$-Gal-Cer (see SI).

The sphingomyelin derivatives were prepared from Azo-THP-Cer, which was phosphorylated using 2-cyanoethyl- $N, N, N^{\prime}, N^{\prime}$-tetraisopropylphosphorodiamidite and $1 \mathrm{H}$-tetrazole, followed by reaction with choline tosylate (see SI). An oxidation directly yielded Azo-THP-SM. Finally, deprotection under acidic conditions gave the unprotected Azo-SM (see SI).

\subsection{Light-responsiveness of membrane-embedded azo-sphingolipids}

Next, we incorporated our newly synthesized Azo- $\beta$-Gal-Cer, Azo-SM, Azo-PhCer, AzoTHP-SM and Azo-THP-Cer photoswitchable lipids (or simply photolipids), as well as Azo-Cer and Azo- $\alpha-G a l-P h C e r$, into raft-mimicking $L_{d}-L_{o}$ phase-separated mixtures containing DOPC, Chol and SM (18:0-SM) and formed small unilamellar vesicles (SUVs) as previously described ${ }^{[36,48]}$. Unless otherwise stated, a molar ratio of 10:6.7:5:5 (DOPC:Chol:SM:photolipid) was typically chosen.

We started by collecting UV-Vis spectra of those various SUV suspensions and characterized the photodynamic properties of the different azobenzene-modified sphingolipids within a membrane environment. As seen in Figs. $1 \mathrm{C}$ and $\mathrm{S1}$ (see SI), all azo-sphingolipids (independently of the headgroup and backbone type) displayed an absorbance maximum, $\lambda_{\max }$, at $\sim 350 \mathrm{~nm}$, when in the dark-adapted state prior irradiation with UV-A or blue light. This peak corresponds to the $\pi \rightarrow \pi^{*}$ transition and is characteristic for the trans-azobenzene isoform. First illumination with UV-A light $(\lambda=365 \mathrm{~nm})$ led to the reduction of the abovementioned absorbance peak, and appearance of a new $\lambda_{\max }$ at $\sim 450 \mathrm{~nm}$. This new peak corresponds to the $n \rightarrow \pi^{*}$ transition and is characteristic for the cis-azobenzene isoform. 
Subsequent irradiation with blue light $(\lambda=470 \mathrm{~nm})$ led then to a back-isomerization of the $N$ acyl azobenzene moieties into the trans-isoform, as confirmed by the disappearance of the absorbance peak at $\sim 450 \mathrm{~nm}$ and reemergence of the absorbance peak at $\sim 350 \mathrm{~nm}$.

Our results indicate that all tested photoswitchable sphingolipids are in the trans-configuration for the dark- and blue light-adapted states, and mostly in cis-configuration for the UV lightadapted state. Similarly, no significant spectral shifts from the characteristic $350 \mathrm{~nm}$ and $450 \mathrm{~nm}$ excitation peaks were observed, pointing out that headgroup functionality and sphingoid base polarity do not critically interfere with the photodynamic properties of the photoswitch.

Subsequently, we deposited SUVs composed of quaternary DOPC:Chol:SM:photolipid mixtures doped with $0.1 \mathrm{~mol} \%$ Atto655-DOPE (dye labelling the $L_{d}$ regions) on top of freshlycleaved mica, to form supported lipid bilayers (SLBs) via vesicle fusion. By collecting fluorescence confocal images, we first evaluated membrane integrity and presence of phaseseparation for samples having sphingosine- (Azo-Cer, Azo- $\beta-G a l C e r, A z o-S M$ ), phytosphingosine- (Azo-PhCer, Azo- $\alpha$-GalPhCer) and blocked THP-sphingosine-based (AzoTHP-SM) lipids. As seen in Figs. S2 and S3, all tested SLBs displayed $L_{d}-L_{\circ}$ phase-separation at the dark-adapted state, with micron-sized rigid $L_{\circ}$ domains (dark areas in fluorescence images) segregated within a fluid $L_{d}$ matrix (red areas in fluorescence images).

The photo-responsiveness and ability of the azo-sphingolipids to then remodel/reorganize phase-separated SLBs were further assessed directly after irradiation with UV-A light $(\lambda=$ $365 \mathrm{~nm}$ ). For membranes lacking photoswitchable lipid (control sample with a DOPC:Chol:SM composition at molar ratio 10:6.7:10), no light-induced remodeling was observed (Fig. S2A). In contrast, for SLBs containing azo-sphingolipids, stark lipid rearrangement dependent on the amount of photolipid present was reported. Here, lipid bilayers with the highest amount of azosphingolipid tested (18.7 mol\%; DOPC:Chol:SM:photolipid with molar ratio 10:6.7:5:5) showed strong reorganization of the $L_{o}$ domains with admixing of fluorescently-marked $L_{d}$ lipids and blurring of the domain boundaries directly after UV-A irradiation (Fig. S2B-G). Intermediate amounts of photoswitchable lipid (11.2 mol\%; DOPC:Chol:SM:photolipid with molar ratio 10:6.7:7:3) led to a significantly lower domain remodeling activity. Finally, for SLBs with the lowest amount of azo-sphingolipid tested (3.7 mol\%; DOPC:Chol:SM:photolipid with molar ratio 10:6.7:5:1) no clearly perceptible membrane reorganization, such as admixing of $L_{d}-L_{o}$ domains, was observed (Fig. S3).

\subsection{Remodeling of membrane domains by sphingosine-based azo-sphingolipids}

After the abovementioned initial characterization, we systematically investigated the lightinduced remodeling of phase-separated DOPC:Chol:SM:photolipid supported membranes (at molar ratio 10:6.7:5:5, meaning 18.7 mol\% photolipid) using high-speed AFM. This technique enables us to capture minor dynamic changes in membrane architecture very accurately, due to its exquisite sub-nm resolution. Herein, we started by analyzing bilayers containing the sphingosine-based photoswitchable lipids Azo-Cer, Azo- $\beta-$ GalCer and Azo-SM.

At the dark-adapted state, those various membranes displayed a height mismatch between the $L_{d}$ and $L_{o}$ regions of $1.1-1.7 \mathrm{~nm}$; similarly to what we reported before for phase-separated bilayers with Azo-Cer (ACes) lipids ${ }^{[36]}$. 
A
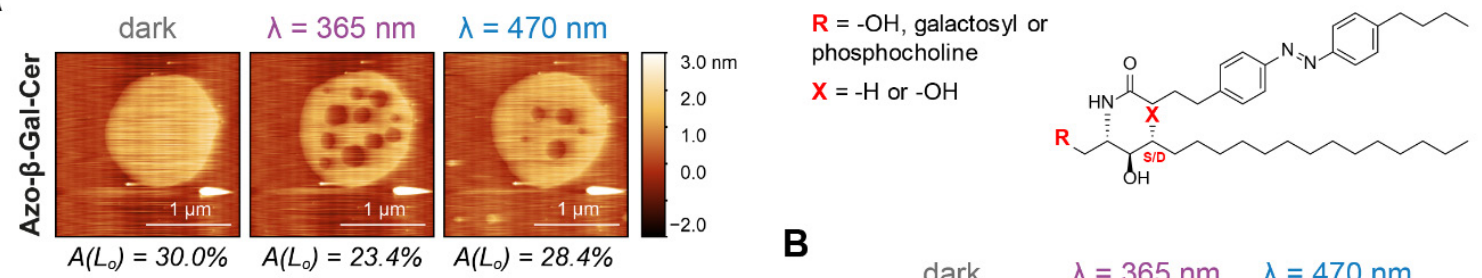

B
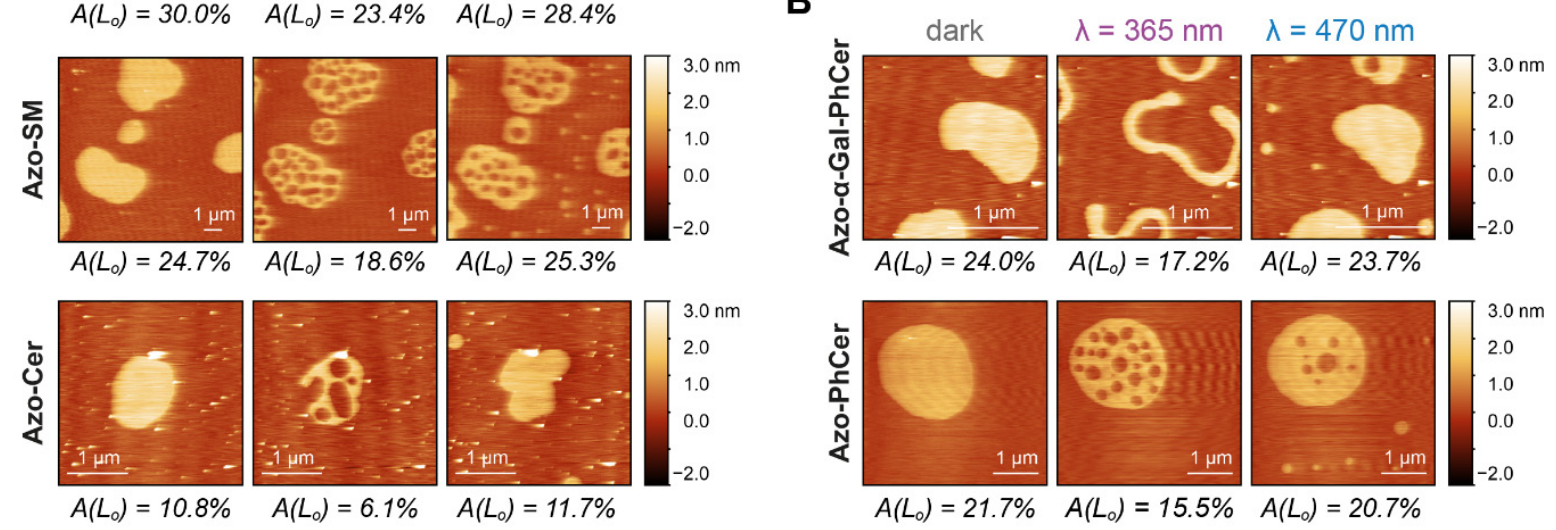

C

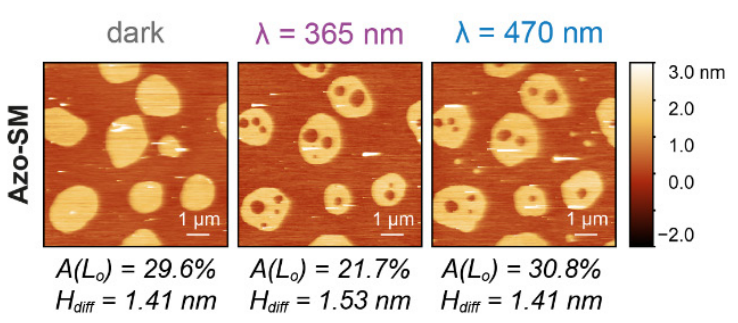

D

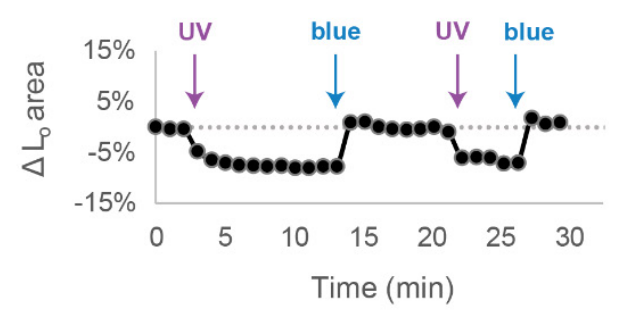

Fig. 2 - Lateral remodeling of phase-separated membranes containing non-blocked azosphingolipids upon light trigger analyzed by high-speed AFM. (A-B) Changes in the area of $L_{0}$ domains before/after illumination with UV-A $(\lambda=365 \mathrm{~nm})$ and blue $(\lambda=470 \mathrm{~nm})$ lights on DOPC:Chol:SM:photolipid (10:6.7:5:5 mol ratio) SLBs having: (A) Azo- $\beta$-Gal-Cer, Azo-SM or Azo-Cer with a sphingosine backbone $(X=-H)$ and varying headgroup functionality $(R=$ galactosyl, phosphocholine or -OH, respectively); (B) Azo- $\alpha$-Gal-PhCer or Azo-PhCer with a phytosphingosine backbone $(X=-\mathrm{OH})$ and varying headgroup functionality $(R=$ galactosyl or $-\mathrm{OH}$, respectively). (C-D) Reversible lateral remodeling of a phase-separated SLB containing Azo-SM, upon UV-A/blue light irradiation, as seen in Movie S4: (C) AFM images of the SLB at the dark-, UV- and blue light-adapted states, displaying the area occupied by the $L_{o}$ phase and the $L_{d}-L_{o}$ height mismatches. (D) Relative variation of total $L_{o}$ area of the SLB over time, shown in Movie S4, upon shining short pulses (marked with arrows) of UV-A and blue light.

Application of UV-A light $(\lambda=365 \mathrm{~nm})$ to bilayers having Azo-Cer, Azo- $\beta$-GalCer or Azo-SM led then to the generation of $L_{d}$ phase, with no major alteration of the $L_{d}$ - $L_{0}$ domain height mismatch (i.e. minor increase of $\sim 0.1 \mathrm{~nm}$ ). As seen in Figs. 2A, S4B and Movies S1-S4, the $\mathrm{UV}$-induced isomerization of the $\mathrm{N}$-acyl chains from a straight trans-form into a kinked cisform, promoted an apparent "fluidization" of the phase-separated membranes, with an overall decrease of the total $L_{o}$ area by $\sim 25 \%$ (area $L_{o}(U V) /$ area $L_{o}($ dark $)=0.75 \pm 0.14$ ), as depicted in Fig. S5. Directly after irradiation with UV-A light, small $L_{d}$ "lakes" were formed within the more rigid thicker $L_{o}$ domains on phase-separated membranes containing Azo-Cer, Azo- $\beta$ GalCer or Azo-SM. The number of fluid $L_{d}$ "lakes" then rapidly dropped, in order to reduce surface tension. While the majority of the smaller $L_{d}$ "lakes" seem to vanish towards the outer fluid $L_{d}$ matrix, few larger fluid $L_{d}$ "lakes" remained trapped inside the rigid $L_{o}$ domains, appearing to grow primarily via Ostwald ripening ${ }^{[49]}$. 
After few minutes of equilibration, subsequent application of a brief pulse of blue light $(\lambda=$ $470 \mathrm{~nm}$ ) to those phase-separated membranes reversed the effect, with $L_{\circ}$ phase being generated. Here, back-isomerization of the $\mathrm{N}$-acyl chains from a kinked cis-form into a straight trans-form, stimulated by blue light, promoted a rigidification of the membranes, with an increase of the total $L_{\circ}$ area back to its original equilibrium dark-adapted value (area $L_{\circ}$ (blue) I area $L_{o}($ dark $\left.)=1.01 \pm 0.18\right)$, as seen in Figs. 2A, S4B and S5. More specifically, upon irradiation with blue light, small rigid $L_{o}$ "islands" were firstly formed within the fluid $L_{d}$ matrix (Movies S1-S4). These taller $L_{\circ}$ "islands" then vanished, as pre-existing $L_{\circ}$ domains grew primarily via Ostwald ripening and domain fusion. Moreover, $L_{\circ}$ domains displayed height values similar to the ones reported before for the initial dark-adapted state. Interestingly, those changes could be repeated over multiple cycles without dissipation effects, with the amount of $L_{d}-L_{o}$ phase-separation alternating between two defined steady-states (or area levels) (Fig. 2C-D).

Besides changes in $L_{d}-L_{\circ}$ phase-separation, we also observed sporadic generation of holes on our supported bilayers after the blue light-triggered conversion of the azo-sphingolipids' $\mathrm{N}$ acyl chains from cis to trans (Fig. S6A). The presence of holes allowed us to recover the total membrane thickness, which was $\sim 5.2 \mathrm{~nm}\left(L_{d}\right.$ thickness $\sim 3.9 \mathrm{~nm}$; Fig. S6B-C); in agreement with previously reported values for membranes of similar lipid composition ${ }^{[50-52]}$.

In summary, all the tested azo-sphingolipids with a sphingosine backbone display a similar photoswitching profile, independently of the type of headgroup. These lipids are able to increase the amount of $L_{d}$ phase on phase-separated membranes upon conversion to the cisisoform after UV-A light irradiation and increase the amount of $L_{\circ}$ phase upon conversion to the trans-isoform after irradiation with blue light.

\subsection{Remodeling of membrane domains by phytosphingosine-based azo-sphingolipids}

Next, we recapitulated the same high-speed AFM procedures on membranes with photoswitchable phytosphingosine-based sphingolipids displaying two hydroxyl groups (3-OH $+4-\mathrm{OH})$ on the phytosphingosine backbone. More precisely, we investigated the ability of these photolipids to interfere with the $L_{d}-L_{\circ}$ phase-separation on supported lipid bilayers, when compared to sphingosine-based lipids having only one hydroxyl $(3-\mathrm{OH})$ on their backbone.

In the dark-adapted state, phase-separated DOPC:Chol:SM:photolipid SLBs with Azo-PhCer and Azo- $\alpha-G a l-P h C e r$ exhibited a domain height mismatch of $1.2-1.8 \mathrm{~nm}$ (Figs. 2B and $S 4 C$ ), very close to the $L_{d}-L_{o}$ height differences here reported for membranes with sphingosine analogs (Figs. 2A and S4B). Upon photoactivation, the $L_{d}-L_{o}$ phase-separated SLBs

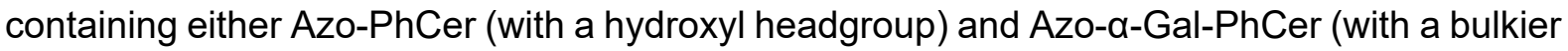
galactosyl headgroup) behaved in a similar way to membranes with sphingosine-based azosphingolipids: exhibiting at the end an identical phenotype of membrane remodeling.

As seen in Figs. 2B, S4C and Movies S5-S6, after irradiation with UV-A light $(\lambda=365 \mathrm{~nm})$, $L_{d}$ "lakes" initially appeared inside existing $L_{o}$ domains, and the total $L_{d}$ phase membrane area lowered by $\sim 23 \%$ (area $L_{\circ}(U V) /$ area $L_{\circ}($ dark $)=0.77 \pm 0.31$, Fig. S5); while the domain height mismatch did not change majorly. Then, after irradiation with blue light $(\lambda=470 \mathrm{~nm}), L_{\circ}$ "islands" initially formed inside the $L_{d}$ regions, and the total $L_{\circ}$ phase membrane area subsequently increased to the initial equilibrium dark-adapted values (area $L_{\circ}$ (blue) / area $\mathrm{L}_{\mathrm{o}}($ dark $)=1.10 \pm 0.36$, Fig. S5). 
Our results confirm that the bulkiness of the neutral headgroup does not play a role in the membrane remodeling ability of our photoswitchable phytosphingolipids. Moreover, the increased backbone polarity of the phytosphingosine backbone does not seem to affect the way azo-phytosphingolipids engage in interactions with their neighboring lipids when compared to azo-sphingolipids. Hereby, we conclude that all the tested Azo-Cer, Azo- $\beta$ GalCer, Azo-SM, Azo- $\alpha-G a l-P h C e r$ and Azo-PhCer establish stable interactions with other sphingolipids (such as SM) and sterols (such as Chol) inside $L_{o}$ domains when their azobenzene acyl chain is in the trans-isoform (imitating a "straight" saturated acyl chain), and with unsaturated phosphatidylcholines (e.g. DOPC) inside $L_{d}$ regions when the azobenzene is in the cis-isoform (mimicking a "bent" unsaturated chain).

\subsection{Remodeling of membrane domains by $3-\mathrm{OH}-$ blocked azo-sphingolipids}

In order to infer the exact role of $\mathrm{H}$-bonding and sphingoid base polarity for the mode of action of azo-sphingolipids, we used high-speed AFM to further investigate the photoswitching and lateral membrane remodeling activities of the azo-sphingolipids Azo-THP-Cer and Azo-THP$\mathrm{SM}$. These lipids have the 3-OH group on their sphingosine backbone protected with a bulky THP moiety. Noteably, the final protecting step resulted in an inseperable mixture of diastereomers at the THP linkage (see Fig. 3), which was used in all experiments.
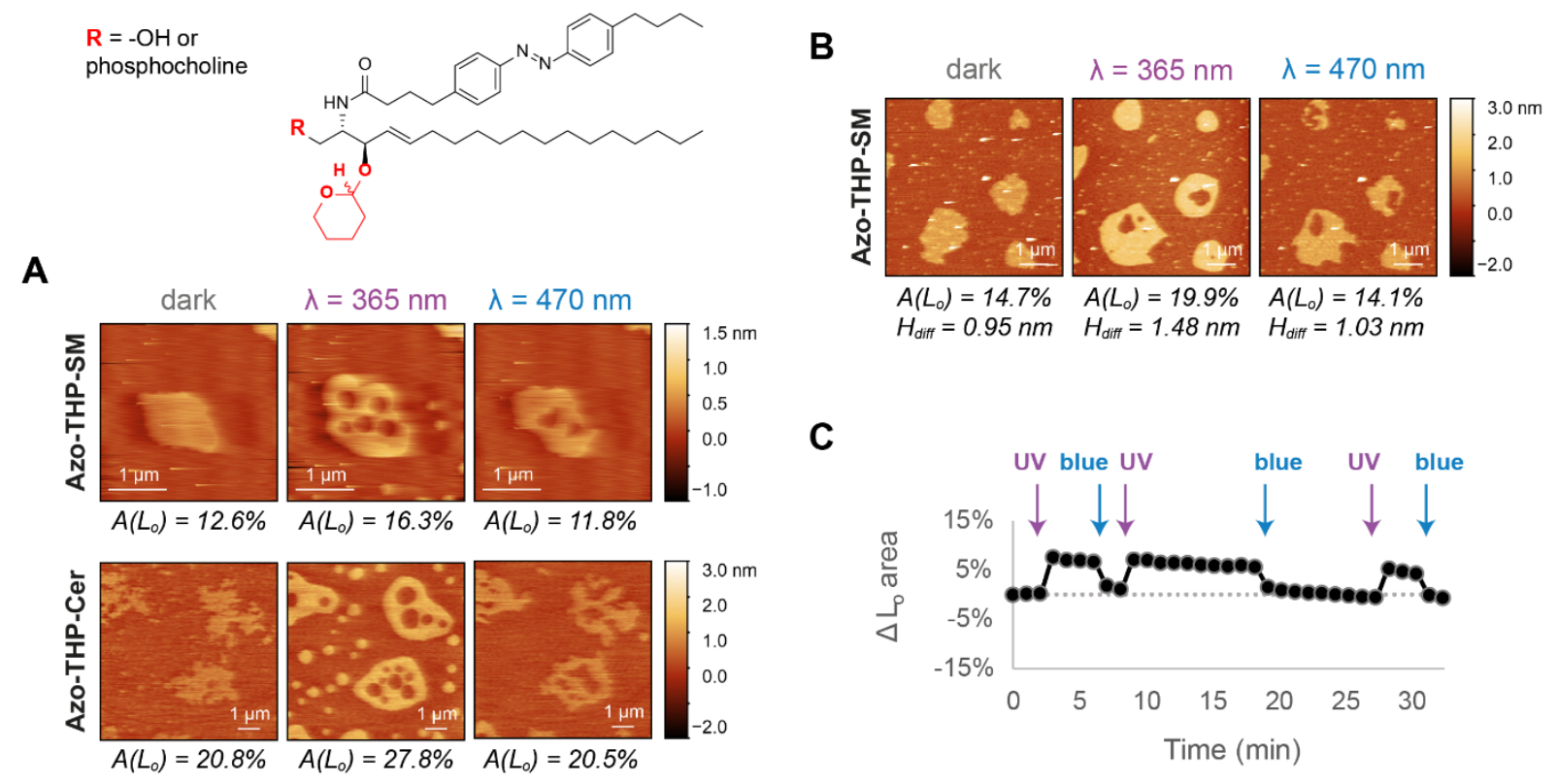

Fig. 3 - Lateral remodeling of phase-separated membranes containing 3-OH-blocked azosphingolipids upon light trigger analyzed by high-speed AFM. (A) Changes in the area of $L_{0}$ domains before/after illumination with UV-A $(\lambda=365 \mathrm{~nm})$ and blue $(\lambda=470 \mathrm{~nm})$ lights on DOPC:Chol:SM:photolipid (10:6.7:5:5 mol ratio) SLBs having Azo-THP-SM or Azo-THP-Cer with a 3$\mathrm{OH}$-blocked (THP-protected) sphingosine backbone and varying headgroup functionality $(R=$ phosphocholine or $-\mathrm{OH}$, respectively). (C-D) Reversible lateral remodeling of a phase-separated SLB containing Azo-THP-SM, upon UV-A/blue light irradiation, as seen in Movie S9: (C) AFM images of the $S L B$ at the dark-, UV- and blue light-adapted states, displaying the area occupied by the Lo phase and the $L_{d}-L_{o}$ height mismatches. (D) Relative variation of total $L_{o}$ area of the SLB over time, shown in Movie S9, upon shining short pulses (marked with arrows) of UV-A and blue light. 
In the dark-adapted trans-form, DOPC:Chol:SM:photolipid bilayers containing 3-OH-blocked Azo-THP-SM or Azo-THP-Cer (Figs. 3, S4D and Movies S7-S9) had $L_{\circ}$ domains with irregular borders and lower height $(\sim 0.6-0.9 \mathrm{~nm})$ when compared to SLBs with non-blocked counterparts (Fig. 2). Such a noticeable effect on the global architecture of $L_{o}$ domains is evidence that 3-OH-blocked lipids are able to reduce the molecular packing within the $\mathrm{L}_{\circ}$ phase, as previously reported for 3-OH-blocked stearoyl-SM[26,53].

When the membranes with THP-protected photoswitchable lipids were irradiated with UV-A light $(\lambda=365 \mathrm{~nm})$, and the lipids converted to the cis-isoform, the total area of $L_{o}$ phase markedly increased $\sim 23 \%$ (area $L_{\circ}(U V) /$ area $L_{\circ}($ dark $)=1.23 \pm 0.21$, Fig. S5), with $L_{\circ}$ domains getting larger, rounder and noticeably heigher ( 1.1-1.4 nm) (Fig. 3A-B). Quite strikingly, directly after exposure to UV-A light, $L_{d}$ "lakes" inside pre-existing $L_{o}$ domains, as well $L_{o}$ "islands" within the $L_{d}$ matrix were transiently formed. The pre-existing $L_{o}$ domains then grew in total area, mainly via Ostwald ripening as $L_{o}$ "islands" disappeared, whereas only few larger $L_{d}$ "lakes" appeared at the end trapped inside the enlarged $L_{\circ}$ domains.

Subsequent illumination with blue light $(\lambda=470 \mathrm{~nm})$ led to an overall decrease of both, total $L_{d}-L_{o}$ height mismatch and $L_{o}$ area back to the initial dark-adapted state values (area $L_{\circ}$ (blue) / area $L_{o}($ dark $)=0.98 \pm 0.20$, Fig. S5), as the 3-OH-blocked photoswitchable lipids isomerized back to the trans-isoform. Interestingly, no noticeable formation of large $L_{d}$ "lakes" or $L_{o}$ "islands" was observed here. The $L_{o}$ domains rapidly shrank with their domain borders becoming irregular (less rounded) and more unstable, as large rapid fluctuations were visible (Movies S7-S9). This clearly indicates that the THP-protected azo-sphingolipids, when in the trans-isoform, severely affect line tension of the phase-separated domains. Finally, the reported $L_{o}$ domain height and area changes within the phase-separated bilayers could be repeated over multiple illumination cycles, as seen in Fig. 3C and Movies S7-S9.

To sum up, photoswitchable sphingolipids having their 3-OH sphingoid moiety blocked with a THP group promote a clearly distinct light-induced reorganization of $L_{d^{-}} L_{o}$ phase-separated membranes, when compared to non-blocked counterparts. These blocked lipids are able to significantly increase the percentual amount and height of the $L_{\circ}$ phase upon UV-triggered isomerization to the cis-isoform, and decrease both these parameters upon blue light-triggered isomerization to the trans-isoform.

\subsection{Phase-separation area changes by photoswitchable sphingolipids}

After demonstrating that the various non-blocked vs. hydroxyl-blocked photoswitchable sphingolipids reorganize phase-separated membranes differently, we set out to quantitatively compare the extent by which these lipids alter the total distribution of phase-separation, as well as other structural membrane parameters.

To begin, since AFM only allows us to follow a limited number of $L_{o}$ domains simultaneously, we acquired additional large field-of-view fluorescence confocal images (Figs. S7, S8A and $4 \mathrm{~A}$ ), to obtain better statistics for determining ensemble area values; independent of domain size and number. We analyzed changes in $L_{o}$ total area before/after UV/blue irradiation on

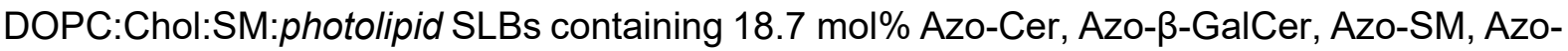
PhCer, Azo-a-GalPhCer, Azo-THP-Cer or Azo-THP-SM, doped with $0.1 \mathrm{~mol} \%$ Atto655-DOPE for fluorescent detection of the $L_{d}$ phase. Usage of fluorescence allowed us to easily generate binary masks (Fig. S7), from which $L_{\circ}$ phase areas could be straightforwardly estimated. 
A

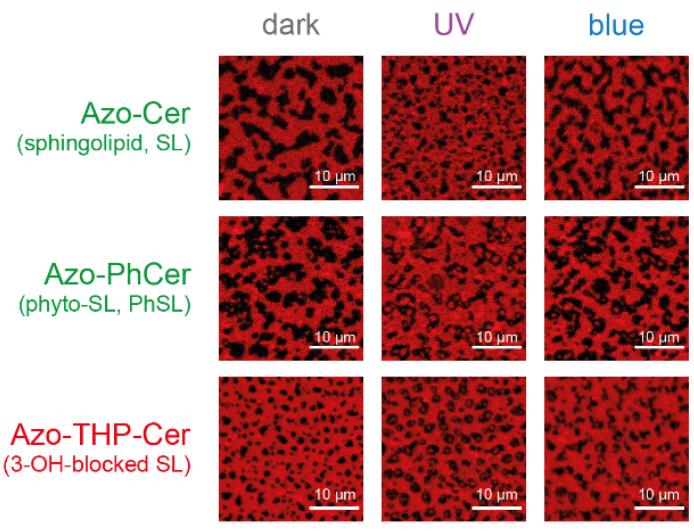

B

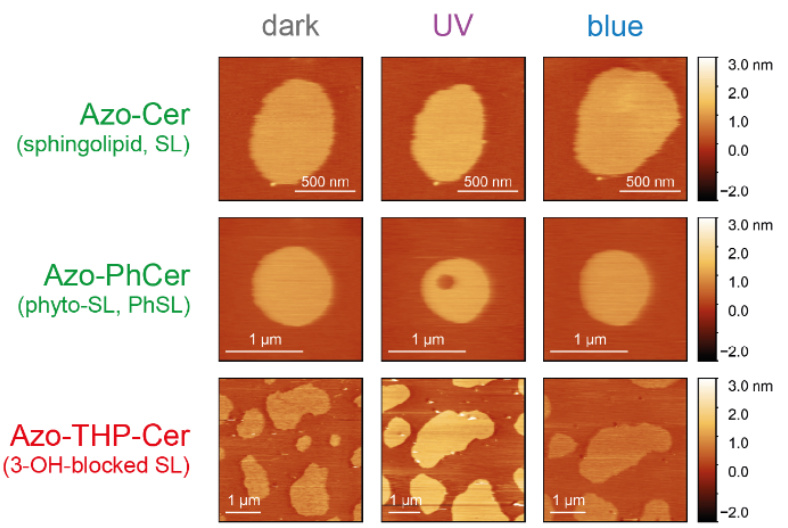

C
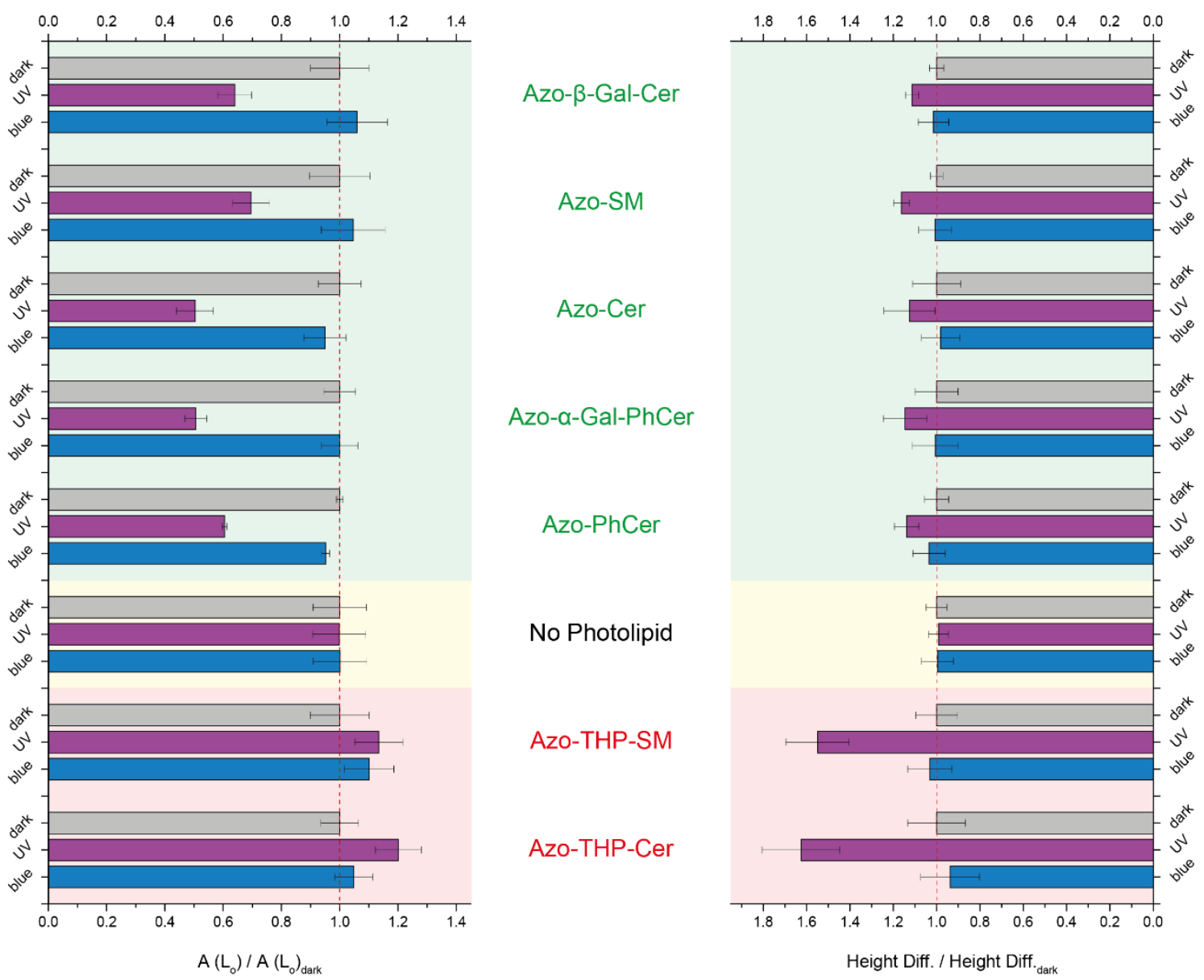

Fig. 4 - Normalized changes in the total $L_{\circ}$ phase area and $L_{d}-L_{\circ}$ height difference on phaseseparated SLBs having different types of azo-sphingolipids, upon application of UV-A $(\lambda=$ $365 \mathrm{~nm})$ and blue $(\lambda=470 \mathrm{~nm})$ lights. Fluorescence confocal $(A)$ and $A F M(B)$ images of DOPC:Chol:SM:photolipid (10:6.7:5:5 mol ratio) SLBs with sphingosine-based Azo-Cer, phytosphingosine-based Azo-PhCer or 3-OH-blocked sphingosine-based Azo-THP-Cer, all having the same $-\mathrm{OH}$ headgroup but distinct sphingoid backbone. Normalized $L_{o}$ areas $(C)$ and $L_{d}$ - $L_{o}$ height differences (D), respectively recovered from fluorescence confocal and AFM data, for phase-separated SLBS having either azo-(phyto)sphingolipids with free 3-OH (marked in green); no azo-sphingolipid (controls with SM, marked in yellow); or THP-protected azo-sphingolipids with the 3-OH blocked (marked in red). Error bars correspond to standard error of the mean $(n=5-9)$. 
To simplify data comparison, the $L_{\circ}$ areas were then normalized before/after UV/blue light illumination by the average $L_{0}$ area for the various individual SLBs at the dark-adapted state (Fig. 4C). In addition, all non-normalized average $L_{o}$ area values recovered for the various SLBs are represented in Fig. S8B.

As seen in Figs. $4 \mathrm{C}$ and $\mathrm{S} 8 \mathrm{~B}$, the total $\mathrm{L}_{\circ}$ area for phase-separated SLBs having sphingosineor phytosphingosine-based photoswitchable lipids decreased in average by $41 \%$ (area $L_{\circ}(U V)$ / area $L_{o}($ dark $\left.)=0.59 \pm 0.04\right)$ after UV-A illumination and augmented back to the original darkadapted state (area $L_{\circ}($ blue $) /$ area $L_{\circ}($ dark $)=1.00 \pm 0.02$ ) upon blue light irradiation. For SLBs having Azo-THP-SM or Azo-THP-Cer, on the contrary, the total area of the $\mathrm{L}_{\circ}$ phase increase by $17 \%$ (area $L_{o}(U V) /$ area $L_{o}($ dark $)=1.17 \pm 0.03$ ) after illumination with UV-A light, while the recorded amount of $L_{\circ}$ phase decreased back to the original dark-adapted state value (area $L_{o}($ blue $) /$ area $L_{o}($ dark $)=1.07 \pm 0.03$ ) after the application of blue light pulse.

Interestingly, if we compare the changes in $\mathrm{L}_{\circ}$ area after azo-sphingolipid photo-isomerization determined by fluorescence (Figs. 4C and S8) vs. high-speed AFM data (Fig. S5), the area changes for confocal microscopy seem to be skewed towards detecting higher amounts of $L_{d}$ phase after UV irradiation. This skew may be a direct consequence of the limited pixel resolution of conventional laser-scanning confocal microscopy for detecting nanoscale $L_{\circ}$ domains, when compared to AFM. Despite this instrumental bias, similar trends in membrane domain area variations were detected with both, fluorescence confocal and AFM techniques.

These experiments corroborate that sphingosine- and phytosphingosine-based photoswitchable lipids rely on the same principles for reshuffling membrane phase-separated domains; whereas the THP-protected counterparts, owing their distinct physicochemical properties, follow a markedly different mechanism.

\subsection{Domain height mismatch changes by photoswitchable sphingolipids}

Our results so far clearly point out that blocking the interfacial hydroxyl on the sphingoid backbone has a marked effect on the molecular organization of individual lipids and on the global architecture of $L_{o}$ domains. Thus, to quantitatively ascertain how photoswitchable sphingolipids affect the structure and physicochemical properties of $L_{0}$ domains within phaseseparated membranes, we collected zoomed-in and high-resolution low-speed AFM images of individual $L_{\circ}$ domains on DOPC:Chol:SM:photolipid SLBs, prior and after illumination with UV-A/blue light, as depicted in Figs. 4B, S9 and S10A. This acquisition mode allows us to follow the membrane contour with an increased signal-to-noise ratio and therefore determine more accurately the height differences between the $L_{o}$ domains and the surrounding $L_{d}$ matrix (Fig. S9). This is an important parameter, as it relates to the hydrophobic mismatch between the saturated ("non-bent" acyl chains) lipids in raft-mimicking $L_{o}$ domains and the unsaturated ("bent" acyl chains) lipids in the more fluid and less packed $L_{d}$ regions.

Altogether, the average height difference between $L_{o}$ and $L_{d}$ regions (at the dark-adapted state for SLBs having either sphingosine- or phytosphingosine-based photolipids was $1.26 \pm 0.11 \mathrm{~nm}$. This value corresponds to the mean of all average $L_{d}-L_{o}$ height differences $( \pm$ standard error) obtained for membranes containing Azo-Cer, Azo- $\beta$-GalCer, Azo-SM, AzoPhCer, Azo- $\alpha-G a l P h C e r$ (Fig. S10B); and was very close to the less precise values previously reported using high-speed AFM. Owing to the exquisite z-resolution of AFM, we also identified that the $L_{o}$ domains of SLBs having azo-sphingolipids with smaller headgroups (e.g. Azo-Cer and Azo-PhCer) were slightly less elevated $(1.04 \pm 0.09 \mathrm{~nm})$ than the $L_{o}$ domains of SLBs 
having azo-sphingolipids with larger headgroups (e.g. Azo-SM, Azo- $\beta$-GalCer and Azo- $\alpha-$ GalPhCer). The later displayed $L_{d}-L_{o}$ height mismatches $(1.41 \pm 0.09 \mathrm{~nm})$ closer to the values recovered $(1.76 \pm 0.05 \mathrm{~nm})$ for control ternary mixtures without photoswitchable lipid (. Hence, our observations corroborate a preferred localization of the trans-azo-sphingolipids inside $\mathrm{L}_{\circ}$ domains, as these lipids could then engage hydrophobic packing and stable $\mathrm{H}$-bonding interactions with $S M$ and $C h o l$, altering slightly the height of $L_{\circ}$ domains due to the different headgroup size and $N$-acyl chain length (e.g. C18:0 acyl chain: 21.2 $\AA$ vs. FAAzo-4: $17.9 \AA$, retrieved from Chem3D, PerkinEImer).

Interestingly, upon applying UV-A light to SLBs having these non-blocked photoswitchable lipids, the $L_{d}$ - $L_{o}$ height mismatch increased in average by $14 \%(1.44 \pm 0.12 \mathrm{~nm}$; Fig. 4D). This is in line with the exclusion of cis-azo-sphingolipids from the $L_{\circ}$ phase and SM being then the predominant sphingolipid molecules inside those domains. Subsequent irradiation with blue light led to the decrease of the domain height by $13 \%$, back to the original values reported for the dark-adapted state (Fig. 4D); corroborating a re-partitioning of trans-azo-sphingolipids back the $L_{\circ}$ phase.

For membranes having hydroxyl-blocked photoswitchable lipids, the height of $L_{\circ}$ domains at the dark-adapted state was lower and the domain boundaries were more irregular, when compared to membranes with non-blocked counterparts. Indeed, the $L_{d}$ - $L_{o}$ height mismatch observed for SLBs with Azo-THP-SM or Azo-THP-Cer was below $1 \mathrm{~nm}(0.73 \pm 0.05 \mathrm{~nm}$; Fig. $\mathrm{S} 10 \mathrm{~B}$ ); similar to the values observed using high-speed AFM, and nearly $0.5 \mathrm{~nm}$ and $1.0 \mathrm{~nm}$ lower than the height mismatch found for SLBs with Azo-SM or control membranes lacking azo-sphingolipids, respectively. The lower $L_{\circ}$ height observed for membranes with 3-OTHPlipids indeed corroborates a preferential localization of these lipids in the $L_{\circ}$ phase when in the trans-isoform, but most importantly validates that blocking $\mathrm{H}$-bonding severely alters inter-lipid interactions, molecular packing, as well line tension within $\mathrm{L}_{o}$ domains.

This destabilization effect can be overcome once the hydroxyl-blocked azo-sphingolipid is converted to its cis-isoform upon illumination with UV-A light. Indeed, after applying UV-A to the phase-separated SLBs with Azo-THP-SM, the $L_{d}-L_{o}$ height mismatch increased by $55 \%$ (Fig. 4D) to average values above $1 \mathrm{~nm}(1.13 \pm 0.07 \mathrm{~nm})$. This elevation in height suggests that Azo-THP-SM and Azo-THP-Cer lipids are expelled from the $L_{\circ}$ phase when in the cisisoform, leaving the $L_{o}$ domains mainly composed by SM and Chol. Without the interference of these THP-protected lipids, SM and Chol molecules can then establish more stable $\mathrm{H}$ bonding and tighter hydrophobic chain packing interactions, giving rise to taller, rounder and larger $L_{0}$ domains. In opposition, irradiation of the phase-separated SLBs with blue light leads to a marked reduction of the $L_{o}$ domain height (Fig. 4D), back to the initial dark-adapted state value $(0.75 \pm 0.05 \mathrm{~nm})$. As the 3-OH-blocked azo-sphingolipids isomerize back to their transisoform, these lipids could then re-establish hydrophobic chain packing interactions with the other raft-localizing, destabilizing the existing $\mathrm{H}$-bonding interactions between SM and Chol.

\subsection{Domain line tension changes by photoswitchable sphingolipids}

A parameter closely linked to the domain height mismatch is line tension, which can be perceived as the interfacial energy arising at the boundaries of coexisting phases and is an important driving force for membrane shape transformation (e.g. budding ${ }^{[4,54,55]}$ and fusion ${ }^{[56]}$ ). In order to estimate the approximate values of line tension for the various raft-mimicking membranes with distinct blocked and non-blocked photoswitchable sphingolipids, based on 
the height mismatch values measured using low-speed AFM, we used the theoretical model implemented by Cohen and coworkers (Eq. 1). This model describes a quadratic dependence of the line tension with the phase height mismatch ${ }^{[57]}$, and was previously used to estimate line tension on phase-separated membranes with similar lipid composition ${ }^{[58]}$.

Overall, as seen in Fig. S10C, line tension values ranged from $1.9-4.3 \mathrm{pN}$ for $L_{d}-L_{o}$ phaseseparated SLBs with sphingosine- and phytosphingosine-based lipids in the trans-isoform. When the lipids are in the cis-isoform and partition to the $L_{d}$ phase instead, a small increase in line tension by $24 \%$ (Fig. S11) was recovered, being the values very close to the ones gauged for phase-separated SLBs lacking azo-sphingolipids $(5.4 \pm 0.6 \mathrm{pN})$.

In contrast, raft-like bilayers with THP-protected azo-sphingolipids (such as Azo-THP-SM and Azo-THP-Cer) in the trans-isoform possess noticeably reduced line tension values $(\sim 1.2 \mathrm{pN})$; $2.2-4.5$-fold lower than the domain line tension measured for SLBs with non-blocked photolipid counterparts (Fig. S10C). Next, when the hydroxyl-blocked lipids are in their cisisoform and locate in the $L_{d}$ phase, the domain line tension greatly increases by $\sim 120 \%$ (Fig. S11), reaching values close to the ones reported for non-blocked counterparts (2.6 pN). Hence, THP-protected photoswitchable lipids, when in trans, greatly reduce the line tension of $L_{o}$ domains in opposition to the azo-sphingolipids with free interfacial hydroxyls, appearing to possess additional line-active (lineactant) properties.

Line-active molecules are known to concentrate at the boundaries of membrane phases ${ }^{[59-62]}$, reducing the hydrophobic mismatch and line tension around phase-separated domains (e.g. $L_{o}$ vs. $L_{d}$ ). Herein, hybrid lipids such as palmitoyl-oleyl-phosphatidylcholine (POPC), possessing both a saturated and unsaturated fatty acid chain, are of particular relevance. When added to ternary mixtures made of lipids with two saturated tails (e.g. DPPC or DSPC), two unsaturated tails (DOPC) and a sterol (Chol), POPC was shown to localize around the borders of the $L_{o}$ phase promoting the reduction of line tension and formation of nanoscopic domains ${ }^{[63-67]}$. Interestingly, Azo-THP-SM and Azo-THP-Cer also appear to reduce line tension and promote the formation of nanoscopic domains in a similar way, sharing moreover key structural similarities with hybrid lipids: a) the trans-azobenzene $\mathrm{N}$-acyl chains mimic saturated fatty acids prone to localize within $L_{o}$ domains, b) the bulky THP moiety at the sphingosine base appears to interfere with the molecular packing of lipids and be therefore susceptible to preferentially localize in the less packed $L_{d}$ phase.

To sum up, the hybrid chain properties in addition to the blockage of $\mathrm{H}$-bonding could be possible explanations for the observed perturbation of the $L_{o}$ domain boundaries and reduction of domain height, by THP-protected azo-sphingolipids in the trans-isoform.

\subsection{Changes in the mechanics (indentation forces) of homogeneous membranes promoted by the photoisomerization of azo-spingolipids}

Despite the differences in membrane domain remodeling by THP-protected vs. non-protected azo-sphingolipids, we also observed the generation of occasional membrane holes when isomerizing 3-OH-blocked photoswitchable lipids from the bent cis-isoform to the straight trans-isoform, as depicted in Fig. S12 for phase-separated membranes with Azo-THP-SM. This hole formation clearly indicates that SLBs containing THP-protected azo-sphingolipids, similarly to membranes with non-blocked counterparts (Fig. S6A), globally expand after UV-A illumination and subsequently compress upon illumination with blue light, due to the bending and unbending on the $\mathrm{N}$-acyl chains respectively. Hence, photoswitching of azo-sphingolipids 
not only influences lipid phase-separation (as discussed so far), but irrefutably affects basic mechanical properties of the membrane, such as packing and stiffness/fluidity.

A

\section{Azo-Cer (sphingolipid, SL)}
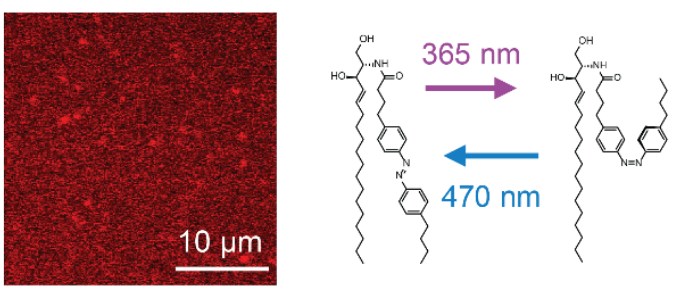

B

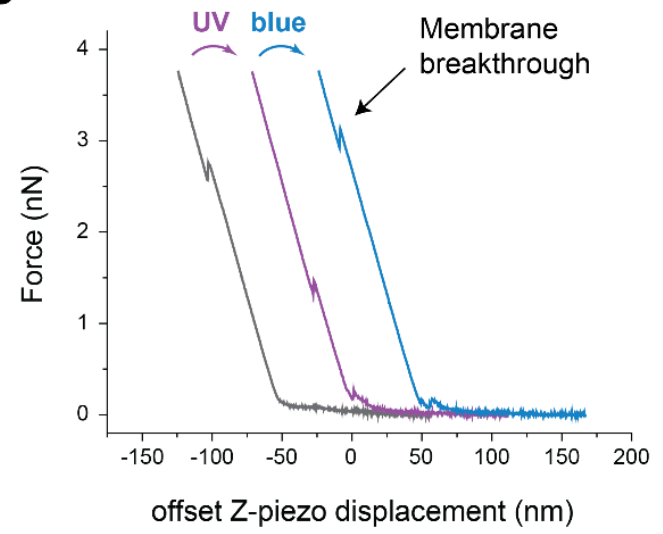

C

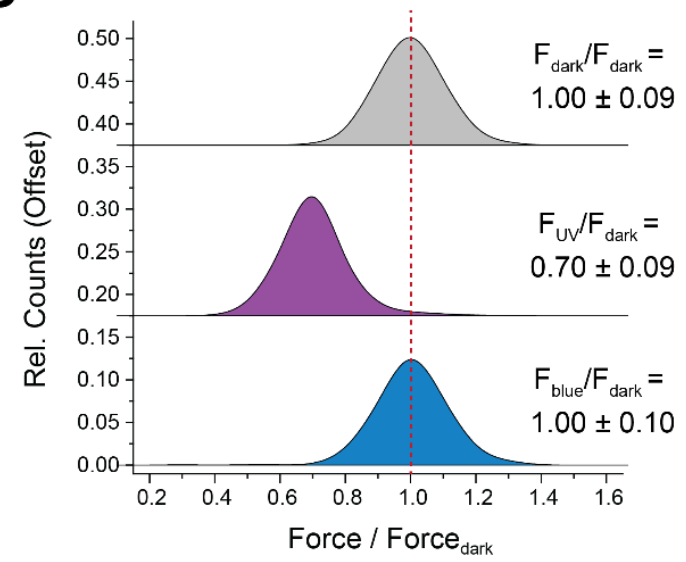

D Azo-THP-SM (3-OH-blocked SL)

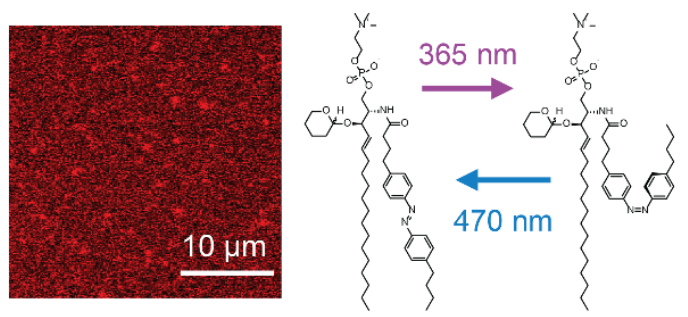

$\mathbf{E}$

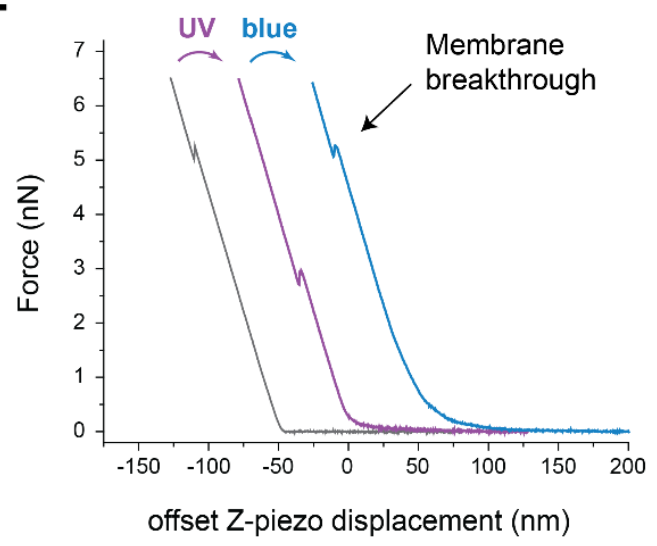

$\mathbf{F}$

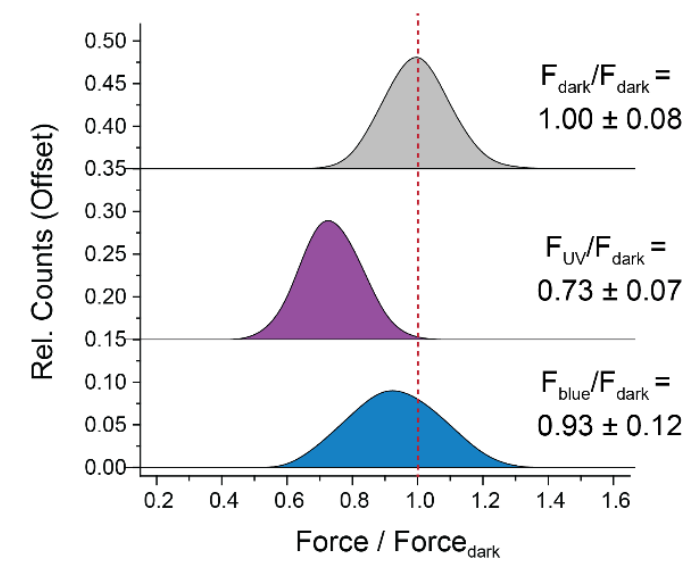

Fig. 5 - Breakthrough forces of homogeneous membranes containing non-blocked Azo-Cer (AC) and 3-OH-blocked Azo-THP-SM (D-F) azo-sphingolipids. (A, D) Confocal images of DOPC:Chol:photolipid (10:6.7:10 mol ratio) supported membranes doped with 0.1 mol\% Atto655-DOPE for fluorescence detection. $(B, E)$ Force spectroscopy indentation curves (Z-piezo displacement) of homogeneous SLBs containing azo-sphingolipids upon illumination with UV-A $(\lambda=365 \mathrm{~nm})$ and blue $(\lambda$ $=470 \mathrm{~nm}$ ) lights. Characteristic membrane breakthrough events for the AFM tip pinching through the SLB marked with arrows. (C, F) Membrane breakthrough histograms normalized to the dark-adapted state for SLBs containing Azo-Cer or Azo-THP-SM prior and after irradiation with UV-A and blue lights. 
In order to evaluate whether photoswitchable sphingolipids indeed interfere with global membrane mechanics, irrespectively of phase-separation, we performed additional AFMbased force spectroscopy measurements ${ }^{[68-75]}$ on non-phase-separated SLBs composed of DOPC:Chol:photolipid (10:6.7:10 molar ratio). More precisely, we evaluated homogenous bilayers containing either Azo-Cer as non-blocked azo-sphingolipid (Fig. 5A), or Azo-THP-SM as 3-OH-blocked azo-sphingolipids (Fig. 5D). In short, when indenting the membranes with an AFM tip, a typical jump (or discontinuity) corresponding to the force required to pierce (or break through) the supported lipid bilayer can be easily identified within the collected forcedisplacement curves (as seen in Fig. 5B,E). The extent of such breakthrough forces is then directly linked to the mechanical properties of the membrane: lower forces are expected when the membranes are more fluid (or less compact); higher forces when these are stiffer (or more compact). Thus, upon recording a set of force curves prior and after illumination with UV-A ( $\lambda$ $=365 \mathrm{~nm})$ and blue $(\lambda=470 \mathrm{~nm})$ lights, we evaluated the breakthrough events and displayed the recovered forces needed to pierce the membranes (Fig. 5C,F) as histograms (nonnormalized values depicted in Fig. S13).

For homogenous DOPC:Chol:Azo-Cer SLBs, a breakthrough force of $2.93 \pm 0.26 \mathrm{nN}$ (Fig. $\mathrm{S} 13 \mathrm{~A}$ ) was recorded in the dark-adapted state. After illumination with UV light, and consequent conversion of Azo-Cer to its bent cis-isoform, the force required for piercing the membrane reduced by $\sim 30 \%$ (Fig. 5C), to $2.06 \pm 0.27 \mathrm{nN}$ (Fig. S13B). Irradiation with blue light, on the contrary, promoted an increase of the breakthrough force, back to its original average value $(2.93 \pm 0.28 \mathrm{nN}$; Fig. S13C), as Azo-Cer would back-isomerize to its trans-isoform. Thus, for non-blocked azo-sphingolipids, we confirmed that the cis-isoform expands/fluidifies the membrane, while trans-isoform compacts/stiffens it. In this context, our force data clearly backs up a previous study ${ }^{[41]}$ based on fluorescence recovery after photobleaching (FRAP), which showed that lateral lipid diffusion on a membrane made of Azo-PC (phosphatidylcholine analog with a FAAzo-4 acyl chain) was higher when the photoswitchable lipid was in its cisisoform and slower when in the trans-isoform.

Likewise, for non-phase-separated DOPC:Chol:Azo-THP-SM bilayers, similar trends were recorded. More precisely, the piercing force needed to break through those membranes also reduced by $\sim 30 \%$ (Fig. 5F), from $4.85 \pm 0.39 \mathrm{nN}$ (Fig. S13D) to $3.56 \pm 0.34 \mathrm{nN}$ (Fig. S13E), upon irradiation with UV light and formation of cis-Azo-THP-SM; in agreement with a global expansion or fluidification of the membrane. Subsequently, the membrane breakthrough force also reverted back close to the original value $(4.51 \pm 0.60 \mathrm{nN}$; Fig. S13F) upon illumination with blue light and formation of trans-Azo-THP-SM: in agreement with a global compaction or rigidification of those membranes.

Therefore, based on this force spectroscopy outcome for homogenous membranes, we can argue that the opposite changes in $\mathrm{L}_{\circ}$ area for phase-separated SLBs containing either 3-OHblocked and non-blocked azo-sphingolipids (observed throughout the previous manuscript sections) are mainly due to different types of interactions these photoswitchable lipids engage via their sphingoid backbone with neighboring lipids within $L_{\circ}$ domains, and are not directly linked to the structural properties of the $N$-acyl photoswitch per se.

\section{Conclusions}

In this work, we evaluated physicochemical foundations for the membrane remodeling ability by a family of photoswitchable sphingolipids, deciphering the relative contributions of the lipid 
headgroup and sphingoid backbone. We synthesized new types of $N$-acyl azobenzene sphingolipids with varying headgroup and sphingoid base functionalities. Then, we studied with the help of atomic force and fluorescence microscopies the propensity of these photoswitchable lipids to alter membrane properties and laterally remodel $L_{d}-L_{\circ}$ phaseseparated supported membranes. Overall, we demonstrated that the headgroup type (simple hydroxyl vs. more complex galactosyl or phosphocholine) does not interfere with the photoswitching ability of the various azo-sphingolipids within raft-mimicking lipid mixtures. Owing to the photo-dynamic reversibility of the azobenzene $\mathrm{N}$-acyl chain, we further highlighted that trans-photolipids (i.e. dark-adapted and blue light-illuminated states) predominantly localize within pre-existing raft-like $L_{\circ}$ domains and compact membranes, while cis-photolipids (i.e. UV-A-illuminated state) preferentially locate within the more fluid $L_{d}$ membrane regions and expand membranes.

Importantly, our results provide clear evidence that the nature of the sphingoid backbone, and their ability for engaging stable $\mathrm{H}$-bonding interactions with other co-lipids, play a fundamental role in the way photoswitchable sphingolipids remodel $L_{d}-L_{o}$ phase-separated membranes and change the amount, size and height of $L_{\circ}$ domains. Sphingosine- and phytospingosine-based lipids, with their free interfacial $3-\mathrm{OH}$ and $4-\mathrm{OH}$ hydroxyls, do not significantly alter the height of $\mathrm{L}_{o}$ domains when in the trans-isoform. In contrast, THP-protected lipids, with their interfacial $3-\mathrm{OH}$ blocked, greatly interfere with the molecular packing and line tension of $\mathrm{L}_{\circ}$ domains, markedly reducing the overall $L_{\circ}$ height mismatch. Whereas non-blocked azo-sphingolipids will promote a decrease of the total $\mathrm{L}_{\circ}$ phase area upon UV trigger, THP-protected azosphingolipids will increase the total $L_{o}$ area, as well as induce a marked rise in $L_{o}$ domain height after illumination with UV light.

Taken together, the structural diversity of the photoswitchable sphingolipids presented here, as well as exquisite understanding of how these lipids alter important membrane properties, may offer new strategies for controlling the structure of biological lipid bilayers and the localization of membrane-interacting proteins. Thus, by further expanding the headgroup repertoire of photoswitchable lipids, we may soon be in the position to target the fate of biologically-relevant proteins on membranes using light as trigger. Such endeavor would not only open up new exciting avenues for optodynamic applications in the fields of synthetic biology, structural biology or biophysics, but also offer novel perspectives towards the development of innovative photo-responsive drugs and pharmacological therapies.

\section{Experimental section}

\subsection{Synthesis of $\mathbf{N}$-acyl azobenzene-modified sphingolipids}

A protocol for the synthesis and analysis of all photolipids can be found in the supporting information (SI).

\subsection{Membrane model systems}

Throughout this work, small unilamellar vesicles (SUVs) and supported lipid bilayers (SLBs) were used as lipid membrane model systems. These were primarily composed by $\mathrm{N}$-stearoylD-erythro-sphingosylphosphorylcholine (C18-SM, or simply SM), 1,2-dioleoyl-sn-glycero-3phosphocholine (DOPC) and cholesterol (Chol), which were purchased from Avanti Polar Lipids (Alabaster, AL, USA); to which different photoswitchable lipids, notably Azo-Cer, Azo- 


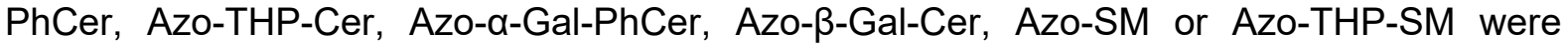
mixed. Unless otherwise stated, the typical lipid composition was DOPC:Chol:SM:photolipid with a 10:6.7:5:5 mol ratio. For fluorescence detected, lipid mixtures were also doped with $0.1 \mathrm{~mol} \%$ Atto655-DOPE, purchased from ATTO Technology GmbH (Siegen, Germany).

SUVs were obtained through bath sonication of multilamellar vesicles. Briefly, the desired lipid mixtures dissolved in choloform:methanol (7:3) were added to a glass vial and the solvent was then evaporated using $\mathrm{N}_{2}$ flow, followed by vacuum-drying in a desiccator. Lipids were rehydrated by adding HEPES buffer (10 mM HEPES, $150 \mathrm{mM} \mathrm{NaCl}, \mathrm{pH} 7.4)$, reaching a final lipid concentration of $10 \mathrm{mM}$, and then vigorously vortexed forming a suspension of multilamellar vesicles. These were then diluted to $1 \mathrm{mM}$ with HEPES buffer, and sonicated in an ultrasonic bath for 10-20 min until the suspension became clear, giving rise to SUVs.

SLBs were prepared by deposition and fusion of SUVs on top of freshly glued-mica glued on a borosilicate coverglass, as described elsewhere ${ }^{[36]}$. Shortly, SUV suspensions (at $1 \mathrm{mM}$ lipid concentration) were deposited in the presence of $2 \mathrm{mM} \mathrm{CaCl} 2$ on freshly-cleaved mica. The samples were then incubated for $20 \mathrm{~min}$ at $65^{\circ} \mathrm{C}$, rinsed with HEPES buffer and allowed to slowly cool down to room temperature for at least $1 \mathrm{~h}$.

\subsection{UV-Vis spectra of membrane-embedded azo-sphingolipids}

UV-Vis spectra of the various azo-sphingolipids embedded within SUVs were collected with Hellma SUPRASIL precision quartz cuvettes $(10 \mathrm{~mm}$ light path) on a Jasco V-650 spectrophotometer (Tokyo, Japan), before and after illumination with UV-A or blue lights. More precisely, SUV suspensions at $150 \mu \mathrm{M}$ lipid concentration, composed of DOPC:Chol:SM:photolipid (10:6.7:7:3 mol ratio) were here utilized.

\subsection{Laser scanning confocal fluorescence microscopy}

Fluorescence microscopy was performed on a Zeiss LSM 510Meta laser scanning microscope (Jena, Germany) using a water immersion objective (C-Apochromat, 40× 1.2W UV-VIS-IR). Samples were excited with the $633 \mathrm{~nm}$ line of a He-Ne laser for Atto655 excitation. Images were typically recorded with 1 Airy unit pinhole and $512 \times 512$ pixel resolution. Image analysis was performed using Fiji software (http://fiji.sc/Fiji).

Segmentation methods: In order to quantify the lipid domain (liquid-ordered phase), the confocal data was processed by a custom-made MATLAB script for batch processing. The algorithm performs basic segmentation operations based on thresholding (Otsu's Method), morphological erosion and dilation operations. The output is an image in Portable Network Graphic format of the positive mask of the dark regions corresponding to the lipid domains and a text file containing the calculated area ratio of the domains for each image.

\subsection{Atomic force microscopy and force spectroscopy}

Atomic force microscopy (AFM) was performed on a JPK Instruments Nanowizard Ultra (Berlin, Germany) mounted on the Zeiss LSM510 Meta laser scanning confocal microscope (Jena, Germany). High-speed and normal-speed AFM, both in AC mode, were done with USCF0.3-k0.3 ultra-short cantilevers from Nanoworld (Neuchâtel, Switzerland) with typical stiffness of $0.3 \mathrm{~N} / \mathrm{m}$. The cantilever oscillation was tuned to a frequency of $100-150 \mathrm{kHz}$ and 
the amplitude was kept below $10 \mathrm{~nm}$. Scan rate was set to $25-150 \mathrm{~Hz}$ for high-speed AFM and to $2-6 \mathrm{~Hz}$ for normal-speed AFM. For both modes, images were acquired with a typical $256 \times 256$-pixel resolution. All measurements were performed at room temperature. The force applied on the sample was minimized by continuously adjusting the set point and gain during imaging. Height, error, deflection and phase-shift signals were recorded and images were linefitted as required. Data was analyzed using JPK data processing software Version 6.0.55 (JPK Instruments) and Gwyddion Version 2.49 (Czech Metrology Institute).

Line tension $(\gamma)$ was determined as previously reported ${ }^{[58]}$ using the equation by Cohen and coworkers ${ }^{[57]}$ :

$\gamma=\frac{\sqrt{B_{S} K_{S} B_{r} K_{S}}}{\sqrt{B_{r} K_{S}}+\sqrt{B_{S} K_{S}}} \cdot \frac{\delta^{2}}{h_{0}^{2}}-\frac{1}{2} \cdot \frac{\left(U_{S} B_{S}-J_{r} B_{r}\right)^{2}}{\sqrt{B_{r} K_{S}}+\sqrt{B_{S} K_{S}}}$

being $\delta$ the $L_{d}-L_{o}$ height mismatch, $\mathrm{h}$ the monolayer thickness with $h_{\circ}=\left(h_{\mathrm{r}}+h_{\mathrm{s}}\right) / 2, B$ the elastic splay modulus, $K$ the tilt modulus, and $J$ the spontaneous curvature of the monolayer. Herein, the subscripts $r$ and $s$ refer to the $L_{o}$ (rigid) and $L_{d}$ (soft) membrane phases, respectively. For calculating the effective heights we used the height mismatches obtained for the various samples and considered a thickness of the $L_{d}$ bilayer of $3.9 \mathrm{~nm}$, as measured in Fig. S6. Finally, as described in García-Sáez et al. ${ }^{[58]}$ we assumed $B_{r}=B_{s}=10 \mathrm{k}_{\mathrm{B}} \mathrm{T}, K_{r}=K_{s}=40 \mathrm{mN} / \mathrm{m}$, and $J_{r}=J_{s}=0$.

Force spectroscopy measurements were performed using uncoated silicon cantilevers CSC38 from MikroMasch (Tallinn, Estonia), with a spring constant of $0.12 \mathrm{~N} / \mathrm{m}$, as previously described ${ }^{[73,74]}$. Shortly, sensitivity and spring constant calibration were done via the thermal noise method. The total z-piezo displacement was then set to $300 \mathrm{~nm}$, indenting approach speed to $800 \mathrm{~nm} / \mathrm{s}$, and the retraction speed was $200 \mathrm{~nm} / \mathrm{s}$, and maximal setpoint to $5-7 \mathrm{nN}$. Force measurements were carried out at different points of the lipid bilayers. Identification of the breakthrough events on an average of 200 approach force curves was done using the JPK data processing software Version 6.0.55 (JPK Instruments), whereas the retrieved yield forces were plotted in histograms using OriginPro2015 (OriginLab).

\subsection{Compound switching on SUVs and SLBs}

Photoswitching of the photolipid compounds was achieved using a CoolLED pE-2 LED light source (Andover, United Kingdom) for illumination at $\lambda=365 \mathrm{~nm}$ and $470 \mathrm{~nm}$. The light source was typically operated for $\sim 20 \mathrm{~s}$ at $80 \%$ power. For the UV-Vis spectroscopic experiments with SUVs inside cuvettes, the light beam was guided by a fiber-optic cable directly to the cuvette top. For microscopic experiments, the light beam was guided by an optical fiber directly through the objective of the LSM510 Meta microscope via a collimator at the backport.

Acknowledgements: The project was funded by the Deutsche Forschungsgemeinschaft (DFG, German Research Foundation) - SFB-1032 - Project ID 201269156. Additional support was provided by the Center for NanoScience (CeNS). H.G.F and P.S acknowledge the financial support by the DFG within the SFB 863 (project ID 111166240). D.T acknowledges the European Research Council (ERC Advanced Grant \#268795, "CARV") and the Deutsche Forschungsgemeinschaft (SFB 749 and CIPSM) for generous funding. N.H. acknowledges financial support by the Deutsche Telekom Foundation and the LMUMentoring program. 
bioRxiv preprint doi: https://doi.org/10.1101/2021.10.11.463883; this version posted October 12, 2021. The copyright holder for this preprint (which was not certified by peer review) is the author/funder. All rights reserved. No reuse allowed without permission.

Further support was given by the Max Planck Society to P.S. The authors thank Sigrid Bauer for assistance in lipid handling and Alena Khmelinskaia for helpful discussions.

Contributions: H.G.F and S.M.L conducted the biophysical experiments and analyzed data. N.H. synthesized and purified most photoswitchable lipids, in cooperation with N.W, H.T-R and J.A.F. P.S and D.T provided funding. D.T supervised the synthesis of photoswitchable lipids and H.G.F their biophysical characterization. H.G.F, N.H and S.M.L prepared the first manuscript draft. All authors contributed to revision of the manuscript.

Conflicts of interest: There are no conflicts of interest.

\section{References}

[1] Y. A. Hannun, L. M. Obeid, Nat. Rev. Mol. Cell Biol. 2018, 19, 175-191.

[2] J. T. Marquês, H. S. Marinho, R. F. M. de Almeida, Prog. Lipid Res. 2018, 71, 18-42.

[3] G. van Meer, D. R. Voelker, G. W. Feigenson, Nat. Rev. Mol. Cell Biol. 2008, 9, 112-124.

[4] T. Baumgart, S. T. Hess, W. W. Webb, Nature 2003, 425, 821-824.

[5] S. L. Veatch, S. L. Keller, Biophys. J. 2003, 85, 3074-3083.

[6] N. Kahya, D. Scherfeld, K. Bacia, B. Poolman, P. Schwille, J. Biol. Chem. 2003, 278, 2810928115.

[7] R. F. M. de Almeida, A. Fedorov, M. Prieto, Biophys. J. 2003, 85, 2406-2416.

[8] F. M. Goñi, A. Alonso, Sphingolipids Apoptosis Dis. 2006, 1758, 1902-1921.

[9] L. C. Silva, R. F. M. de Almeida, B. M. Castro, A. Fedorov, M. Prieto, Biophys. J. 2007, 92, 502516.

[10] S. Chiantia, N. Kahya, P. Schwille, Langmuir 2007, 23, 7659-7665.

[11] B. M. Castro, M. Prieto, L. C. Silva, Prog. Lipid Res. 2014, 54, 53-67.

[12] N. Kondo, Y. Ohno, M. Yamagata, T. Obara, N. Seki, T. Kitamura, T. Naganuma, A. Kihara, Nat. Commun. 2014, 5, 5338.

[13] B. Školová, A. Kováčik, O. Tesař, L. Opálka, K. Vávrová, Biochim. Biophys. Acta BBA Biomembr. 2017, 1859, 824-834.

[14] S. T. Pruett, A. Bushnev, K. Hagedorn, M. Adiga, C. A. Haynes, M. C. Sullards, D. C. Liotta, A. H. Merrill Jr., J. Lipid Res. 2008, 49, 1621-1639.

[15] E. Bieberich, Chem. Phys. Lipids 2018, 216, 114-131.

[16] K. Simons, W. L. C. Vaz, Annu. Rev. Biophys. Biomol. Struct. 2004, 33, 269-295.

[17] E. Sezgin, I. Levental, S. Mayor, C. Eggeling, Nat. Rev. Mol. Cell Biol. 2017, 18, 361-374.

[18] I. Levental, S. L. Veatch, Mol. Biol. Membr. Lipids 2016, 428, 4749-4764.

[19] J. P. Slotte, Biochim. Biophys. Acta BBA - Biomembr. 2016, 1858, 304-310.

[20] S. Chiantia, N. Kahya, P. Schwille, Langmuir 2005, 21, 6317-6323.

[21] S. Chiantia, N. Kahya, J. Ries, P. Schwille, Biophys. J. 2006, 90, 4500-4508.

[22] S. Chiantia, J. Ries, G. Chwastek, D. Carrer, Z. Li, R. Bittman, P. Schwille, Biochim. Biophys. Acta BBA - Biomembr. 2008, 1778, 1356-1364.

[23] H. G. Franquelim, S. Chiantia, A. S. Veiga, N. C. Santos, P. Schwille, M. A. Castanho, Aids 2011, 25, 419-428.

[24] I. Visco, S. Chiantia, P. Schwille, Langmuir 2014, 30, 7475-7484.

[25] J. M. Boggs, Biochim. Biophys. Acta BBA - Rev. Biomembr. 1987, 906, 353-404.

[26] C. C. Kan, Z. S. Ruan, R. Bittman, Biochemistry 1991, 30, 7759-7766.

[27] E. Mombelli, R. Morris, W. Taylor, F. Fraternali, Biophys. J. 2003, 84, 1507-1517.

[28] A. Björkbom, T. Róg, K. Kaszuba, M. Kurita, S. Yamaguchi, M. Lönnfors, T. K. M. Nyholm, I. Vattulainen, S. Katsumura, J. P. Slotte, Biophys. J. 2010, 99, 3300-3308.

[29] D. L. Gater, V. Réat, G. Czaplicki, O. Saurel, A. Milon, F. Jolibois, V. Cherezov, Langmuir 2013, 29, 8031-8038.

[30] I. Artetxe, C. Sergelius, M. Kurita, S. Yamaguchi, S. Katsumura, J. P. Slotte, T. Maula, Biophys. J. 2013, 104, 604-612. 
bioRxiv preprint doi: https://doi.org/10.1101/2021.10.11.463883; this version posted October 12, 2021. The copyright holder for this preprint (which was not certified by peer review) is the author/funder. All rights reserved. No reuse allowed without permission.

[31] S. Guo, T. C. Moore, C. R. lacovella, L. A. Strickland, C. McCabe, J. Chem. Theory Comput. 2013, 9, 5116-5126.

[32] T. Yasuda, M. A. Al Sazzad, N. Z. Jäntti, O. T. Pentikäinen, J. P. Slotte, Biophys. J. 2016, 110, 431-440.

[33] A. Björkbom, T. Róg, P. Kankaanpää, D. Lindroos, K. Kaszuba, M. Kurita, S. Yamaguchi, T. Yamamoto, S. Jaikishan, L. Paavolainen, J. Päivärinne, T. K. M. Nyholm, S. Katsumura, I. Vattulainen, J. P. Slotte, Biochim. Biophys. Acta BBA - Biomembr. 2011, 1808, 1179-1186.

[34] J. A. Frank, M. Moroni, R. Moshourab, M. Sumser, G. R. Lewin, D. Trauner, Nat. Commun. 2015, 6, 7118.

[35] J. Morstein, A. C. Impastato, D. Trauner, ChemBioChem 2021, 22, 73-83.

[36] J. A. Frank, H. G. Franquelim, P. Schwille, D. Trauner, J. Am. Chem. Soc. 2016, 138, $12981-$ 12986.

[37] N. Hartrampf, T. Seki, A. Baumann, P. Watson, N. A. Vepřek, B. E. Hetzler, A. Hoffmann-Röder, M. Tsuji, D. Trauner, Chem. - Eur. J. 2020, 26, 4476-4479.

[38] S. D. Pritzl, P. Urban, A. Prasselsperger, D. B. Konrad, J. A. Frank, D. Trauner, T. Lohmüller, Langmuir 2020, 36, 13509-13515.

[39] J. Morstein, M. Kol, A. J. E. Novak, S. Feng, S. Khayyo, K. Hinnah, N. Li-Purcell, G. Pan, B. M. Williams, H. Riezman, G. E. Atilla-Gokcumen, J. C. M. Holthuis, D. Trauner, ACS Chem. Biol. 2021, DOI 10.1021/acschembio.0c00823.

[40] H. A. Scheidt, K. Kolocaj, D. B. Konrad, J. A. Frank, D. Trauner, D. Langosch, D. Huster, Biochim. Biophys. Acta BBA - Biomembr. 2020, 1862, 183438.

[41] P. Urban, S. D. Pritzl, M. F. Ober, C. F. Dirscherl, C. Pernpeintner, D. B. Konrad, J. A. Frank, D. Trauner, B. Nickel, T. Lohmueller, Langmuir 2020, 36, 2629-2634.

[42] P. Urban, S. D. Pritzl, D. B. Konrad, J. A. Frank, C. Pernpeintner, C. R. Roeske, D. Trauner, T. Lohmüller, Langmuir 2018, 34, 13368-13374.

[43] C. Pernpeintner, J. A. Frank, P. Urban, C. R. Roeske, S. D. Pritzl, D. Trauner, T. Lohmüller, Langmuir 2017, 33, 4083-4089.

[44] J. Holmgren, I. Lönnroth, J. Månsson, L. Svennerholm, Proc. Natl. Acad. Sci. 1975, 72, 2520.

[45] H. Ewers, W. Römer, A. E. Smith, K. Bacia, S. Dmitrieff, W. Chai, R. Mancini, J. Kartenbeck, V. Chambon, L. Berland, A. Oppenheim, G. Schwarzmann, T. Feizi, P. Schwille, P. Sens, A. Helenius, L. Johannes, Nat. Cell Biol. 2010, 12, 11-18.

[46] D. G. Cook, J. Fantini, S. L. Spitalnik, F. Gonzalez-Scarano, Virology 1994, 201, 206-214.

[47] R. R. Schmidt, P. Zimmermann, Angew. Chem. Int. Ed. Engl. 1986, 25, 725-726.

[48] M. Kol, B. Williams, H. Toombs-Ruane, H. G. Franquelim, S. Korneev, C. Schroeer, P. Schwille, D. Trauner, J. C. Holthuis, J. A. Frank, eLife 2019, 8, e43230.

[49] V. A. J. Frolov, Y. A. Chizmadzhev, F. S. Cohen, J. Zimmerberg, Biophys. J. 2006, 91, 189-205.

[50] H. An, M. R. Nussio, M. G. Huson, N. H. Voelcker, J. G. Shapter, Biophys. J. 2010, 99, 834-844.

[51] J. D. AU - Unsay, K. AU - Cosentino, A. J. AU - García-Sáez, J. Vis. Exp. 2015, e52867.

[52] U. Bhojoo, M. Chen, S. Zou, Biochim. Biophys. Acta BBA - Biomembr. 2018, 1860, 700-709.

[53] M. D. Lister, Z. Ruan, R. Bittman, Biochim. Biophys. Acta BBA - Lipids Lipid Metab. 1995, 1256, 25-30.

[54] R. Lipowsky, Biophys. J. 1993, 64, 1133-1138.

[55] D. Vind-Kezunovic, C. H. Nielsen, U. Wojewodzka, R. Gniadecki, Biochim. Biophys. Acta BBA Biomembr. 2008, 1778, 2480-2486.

[56] S.-T. Yang, V. Kiessling, L. K. Tamm, Nat. Commun. 2016, 7, 11401.

[57] P. I. Kuzmin, S. A. Akimov, Y. A. Chizmadzhev, J. Zimmerberg, F. S. Cohen, Biophys. J. 2005, 88, 1120-1133.

[58] A. J. García-Sáez, S. Chiantia, P. Schwille, J. Biol. Chem. 2007, 282, 33537-33544.

[59] L. V. Schäfer, S. J. Marrink, Biophys. J. 2010, 99, L91-L93.

[60] R. Brewster, S. A. Safran, Biophys. J. 2010, 98, L21-L23.

[61] T. Yamamoto, S. A. Safran, Soft Matter 2011, 7, 7021-7033.

[62] Z. Li, A. A. Gorfe, J. Phys. Chem. B 2014, 118, 9028-9036.

[63] F. A. Heberle, R. S. Petruzielo, J. Pan, P. Drazba, N. Kučerka, R. F. Standaert, G. W. Feigenson, J. Katsaras, J. Am. Chem. Soc. 2013, 135, 6853-6859.

[64] E. Hassan-Zadeh, E. Baykal-Caglar, M. Alwarawrah, J. Huang, Langmuir 2014, 30, 1361-1369.

[65] N. Shimokawa, M. Nagata, M. Takagi, Phys. Chem. Chem. Phys. 2015, 17, 20882-20888.

[66] R. D. Usery, T. A. Enoki, S. P. Wickramasinghe, M. D. Weiner, W.-C. Tsai, M. B. Kim, S. Wang, T. L. Torng, D. G. Ackerman, F. A. Heberle, J. Katsaras, G. W. Feigenson, Biophys. J. 2017, $112,1431-1443$.

[67] W.-C. Tsai, G. W. Feigenson, Biochim. Biophys. Acta BBA - Biomembr. 2019, 1861, 478-485. 
bioRxiv preprint doi: https://doi org/10.1101/2021 10.11.463883 : this version posted October 12, 2021. The copyright holder for this preprint (which was not certified by peer review) is the author/funder. All rights reserved. No reuse allowed without permission.

[68] H.-J. Butt, V. Franz, Phys Rev E 2002, 66, 031601.

[69] S. Loi, G. Sun, V. Franz, H.-J. Butt, Phys. Rev. E 2002, 66, 031602.

[70] S. Garcia-Manyes, L. Redondo-Morata, G. Oncins, F. Sanz, J. Am. Chem. Soc. 2010, 132, 12874-12886.

[71] R. M. A. Sullan, J. K. Li, C. Hao, G. C. Walker, S. Zou, Biophys. J. 2010, 99, 507-516.

[72] S. Chiantia, J. Ries, N. Kahya, P. Schwille, ChemPhysChem 2006, 7, 2409-2418.

[73] A. J. García-Sáez, S. Chiantia, J. Salgado, P. Schwille, Biophys. J. 2007, 93, 103-112.

[74] H. G. Franquelim, D. Gaspar, A. S. Veiga, N. C. Santos, M. A. R. B. Castanho, Biochim. Biophys. Acta BBA - Biomembr. 2013, 1828, 1777-1785.

[75] M. M. Domingues, B. Gomes, A. Hollmann, N. C. Santos, Int. J. Mol. Sci. 2021, 22, DOI 10.3390/ijms22052574. 